Submitted to

Physics Letters B

CERN/EP/PHYS 77-56

8 December 1977

\title{
DI-LEPTON EVENTS IN THE BEBC NARROW-BAND BEAM NEUTRINO EXPERIMENT
}

Aachen-Bonn-CERN-London-Oxford-Saclay Collaboration

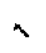

P.C. BOSETTI, H. DEDEN, P. FRITZE, H, GRÄSSLER, F.J. HASERT, D. KIRCH, J. MORFIN, H. SEYFERT, P. SCHMITZ and R. SCHULTE

III. Physikalisches Institut der Technischen Hochschule, Aachen

K. BOCKMANN, H. EMANS, C. GEICH-GIMBEL, R: HARTMANN, A. KELLER, T.P. KOKOTT, W. MEINCKE, B. NELLEN and R. PECH

Physikalisches Institut der Universität Bonn, Bonn

L. BARONE ${ }^{(*)}$, D.C. CUNDY, J. FIGIEL ${ }^{(* *)}$, A. GRANT, D. HAIDT, P.O. HULTH ${ }^{(* * *)}$, D.J. KOCHER ${ }^{(+)}$, D.R.O. MORRISON, E. PAGIOLA, L. PAPE, V. PETERSON $(++)$, CH. PEYROU, P. PORTH ${ }^{(++)}$, P. SCHMID, H. WACHSMUTH and K.L. WERNHARD ${ }^{(+)}$ CERN, European Organization for Nuclear Research, Geneva

S. BANERJEE, K.W.J. BARNHAM, R. BEUSELINCK, I. BUTTERWORTH, E.F. CLAYTON, D.B. MILLER, K.J. POWELL and E.J.W. WEST

Imperial College of Science and Technology, London

C.L. DAVIS, P. GROSSMANN, R. McGOW, J.H. MULVEY, G. MYATT, D.H. PERKINS, R. PONS, D. RADOJICIC, P. RENTON and B. SAITTA

Department of Nuclear Physics, Oxford

V. BARUZZI, M. BLOCH, M. DEBEER, W. HART, Y. SACQUIN, B. TALLINI and T. VIGNAUD

Centre d'Etudes Nucléaires, Saclay

(*) Summer student from the University of Rome

(**) Visiting scientist from Institute of Nuclear Physics, Krakow

(***) CERN Fellow from University of Stockholm

(+) CERN Fellow from University of Innsbruck

$(++)$ Now at the University of Hawaii

(+++) CERN Fellow from the Institut für Hochenergiephysik, Vienna 


\section{ABSTRACT}

In film taken with $\mathrm{BEBC}$ filled with a Ne $(74 \% \mathrm{~mol}) / \mathrm{H}_{2}$ mixture and exposed to the CERN narrow-band neutrino beam $(200 \mathrm{GeV})$, ten $\mu^{-} \mu^{+}$, one $\mu^{-\mu^{+}} \mu^{-}$and five $\mu^{-e^{+}}$events were found in the $\nu$ exposure and two $\mu^{+} \mu^{-}$ events and one $\mu^{+} \mathrm{e}^{-}$event in the $\bar{v}$ exposure. Assuming the same production mechanism for $\mu \mu$ and $\mu$ e events, the relative rates, corrected for identification inefficiency and background, can be combined and are $(1.3 \pm 0.4) \%$ for $\left(\mu^{-} \mathrm{e}^{+}+\mu^{-} \mu^{+}\right) / \mu^{-}$in neutrino interactions, and $(1.2 \pm 0.5) \%$ for $\left(\mu^{+} \mathrm{e}^{-}+\mu^{+} \mu^{-}\right) / \mu^{+}$in antineutrino interactions. Eight out of the sixteen $\mu^{-} l^{+}$events have a $v^{0}$, leading to a corrected $\mathrm{K}^{\circ}-$ multiplicity of $1.7 \pm 0.7$. No evidence is found for heavy lepton production. 
Neutrino interactions leading to the production of two muons of opposite charge were first observed in counter experiments at FNAL [1]. Subsequent1y, the production of electrons $\left(e^{+}\right)$in association with muons $\left(\mu^{-}\right)$by neutrinos was observed at CERN in the Gargamelle bubble chamber [2] and in the 15' bubble chamber at FNAL [3]. Recent1y, a CERN counter experiment recorded and analyzed large numbers of multi-muon events induced by neutrinos and antineutrinos [4]. The bubble chamber experiments found evidence for a strong correlation of di-lepton events with production of strange particles, an association which supports the interpretation [1] of these events as being due to the production and subsequent decay of charmed hadrons in charged current interactions.

In this letter, results are reported of a combined search for di-muon and muon-electron events from an experiment performed in the CERN $3.7 \mathrm{~m}$ bubble chamber ( $B E B C)$. $B E B C$ was filled with a neon-hydrogen mixture (74\% mol neon, radiation length $42 \mathrm{~cm}$ ) and exposed to the CERN SPS narrow-band neutrino and antineutrino beams (200 GeV/c transport momentum). A fiducial volume of $17 \mathrm{~m}^{3}$ (12 tons) was chosen so that the minimum down-stream path from the interaction vertex was $0.5 \mathrm{~m}$. An External Muon Identifier (EMI) [5] was used; it consisted of a single plane formed from 49 proportional wire chambers each of area $3 \times 1 \mathrm{~m}^{2}$ located $\sim 6 \mathrm{~m}$ from the centre of $\mathrm{BEBC}$ and separated from the chamber liquid by $600-2000 \mathrm{~g} / \mathrm{cm}^{2}$ of absorber material.

100000 pictures with neutrinos incident and 180000 pictures with antineutrinos incident have been scanned twice giving a combined scanning efficiency of $98 \%$. Neutrino and antineutrino cross sections from this experiment have been reported previously [6]. The charged current (CC) event sample used in the present di-lepton search consists of $\sim 1000$ neutrino and $\sim 260$ antineutrino events. The muon in these events was identified as a non interacting particle of the right charge $\left(\mu^{-}\right.$in $\nu, \mu^{+}$ in $\bar{v}$ ) leaving the bubble chamber and with momentum greater than $5 \mathrm{GeV} / \mathrm{c}$, which could be extrapolated into the EMI and associated spatially with a hit in the EMI. The detection efficiency for these muons was determined to be 98\%; misidentification of tracks as muons due to spurious association or hadron punch-through is negligible. 
CERN/EP/PHYS 77-56

Di-muon events - A search was made in the CC samples for events having at least one other track of either sign associated to a hit in the EMI in time coincidence ( $\lesssim 500 \mathrm{~ns}$ ) with the hit corresponding to the first muon. The small rate of accidental time coincidences typical of a narrowband beam allowed the cut on the momentum of additional muons to be reduced to $4 \mathrm{GeV} / \mathrm{c}$. With this cut, a geometrical detection efficiency of $95 \%$ is still maintained. Ten events were found with $\mu^{-} \mu^{+}$, one with $\mu^{-} \mu^{+} \mu^{-}$, three with $\mu^{-} \mu^{-}$in the neutrino experiment, and two $\mu^{+} \mu^{-}$events in the antineutrino sample (table 1 ).

The only significant background comes from undetected $\pi_{\mu 2}$ and $k_{\mu 2}$ decays between the event vertex and the EMI. This background was determined from a Monte-Carlo simulation of the hadron spectra $(K / \pi$ ratio taken as 0.1 ) which calculated the potential decay length of all tracks, and the resulting association of the decay particle in the EMI. This calculation yields a background of $2.7 \mu^{-} \mu^{+}$and $1.7 \mu^{-} \mu^{-}$events in the neutrino run and $0.5 \mu^{+} \mu^{-}$and $0.4 \mu^{+} \mu^{+}$events in the antineutrino run. The background estimated for the tri-muon event being a di-muon event with a third muon from a $K_{\mu 2}$ decay (assuming one charged kaon per di-lepton event) is 0.2 events; the tri-muon event is included in the di-muon sample.

Muon-electron events - In addition to the double general scan referred to above, all events in the sample considered here have been reexamined in a search for direct electron production at the interaction vertex; at the same time information was collected on Dalitz pair production, delta-rays, Compton electrons, pair production near the vertex, and the distance of confusion near the vertex, which in some cases may prevent detection of the origin of an electron.

To be accepted as due to a primary electron, a track must fulfill at least one of the following criteria:

(a) a sudden change in curvature, without a visible kink (non-converted bremsstrahlung),

(b) an $\mathrm{e}^{+} \mathrm{e}^{-}$pair pointing tangentially to the track (converted bremsstrahlung), 
CERN/EP/PHYS 77-56

(c) an $\mathrm{e}^{+} \mathrm{e}^{-}$pair lying on the track with more than $10 \%$ of the track energy (direct pair production),

(d) a delta-ray taking at least $50 \%$ of the incident energy.

The criterion of spiralization was not used because of possible confusion with low energy $\pi-\mu-\mathrm{e}$ decays.

An analysis of electron and positron tracks from identified $\gamma \rightarrow \mathrm{e}^{+} \mathrm{e}^{-}-$ pairs shows that in the conditions of this experiment, these criteria provide an electron identification efficiency of $(90 \pm 5) \%$ above $1 \mathrm{GeV}$ up to $\sim 10 \mathrm{GeV}$ electron energy $((85 \pm 5) \%$ between 0.3 and $I \mathrm{GeV})$. From the double scan and special scan, the efficiency for finding prompt electron candidates is estimated to be $(95 \pm 5) \%$.

In addition to satisfying one of the above criteria, the candidates for primary electrons were required to be clearly visible on at least one view as single tracks leaving the vertex; all cases of possible ambiguity with asymmetric Dalitz pairs or other processes, or in which the vertex region was obscured by many close tracks, were rejected.

Five $\mu^{-} \mathrm{e}^{+}$events were found in the neutrino sample, and one $\mu^{+} \mathrm{e}^{-}$ event in the antineutrino sample (table 1 ).

Background for $\mu^{-} e^{+}$events with an identified muon can come from asymmetric Dalitz pairs, $\mathrm{K}_{\mathrm{e}}$ decays in flight without visible kink, $\pi_{\mu 2}^{+}$ decays at rest, and for $\mu^{+} e^{-}$events also from $\delta$-rays and Compton electrons. An electron momentum cut of $0.3 \mathrm{GeV} / \mathrm{c}$ was made to exclude the contribution from $\pi-\mu-e$ decays and reduce the background from asymetric Dalitz pairs, $\gamma$-rays and Compton electrons.

Asymetric Dalitz pairs (including gamma conversions occurring very close to the vertex) with less than $5 \mathrm{MeV} / \mathrm{c}$ in the lower momentum branch (making the track hard to distinguish) and more than $0.3 \mathrm{GeV} / \mathrm{c}$ in the higher momentum branch are estimated to yield 0.3 and 0.1 background events for neutrinos and anti-neutrinos, respectively. This background is significant only for positrons (electrons) below $1 \mathrm{GeV} / \mathrm{c}$. The background from $\mathrm{K}_{\mathrm{e} 3}$ decays is estimated to be $\sim 2 \cdot 10^{-5}$ per CC event. 
An uncertainty in the rate for he events arises from confused vertices. Since the candidate track is required to be clearly visible and the vertex unobscured so that possible asymetric Dalitz partners can be seen, this leads to a loss of events rather than a contribution to background. An analysis of the distribution of the "distance of confusion" about vertices leads to an estimated correction factor for this loss of $1.3 \pm 0.2$ which, together with the electron scanning and identification in efficiencies, leads to an overall correction factor of $1.5 \pm 0.3$.

Results and discussion - Table 1 lists the characteristics of all events found in the fiducial volume with a (right-sign) muon and at least one more lepton and with a total visible energy greater than $10 \mathrm{Gev}$.

The relative rates for di-lepton events, corrected for identification inefficiency and background are shown in table 2. If the $\mu \mu$ and $\mu e$ events have the same production mechanism, the observations can be combined. The results are given in table 2 for a $4 \mathrm{GeV} / \mathrm{c}$ momentum cut for the second lepton (which also reduces the $\mu$ e background to a negligible leve1).

Eight of all the sixteen opposite-sign di-lepton events due to neutrino and one of the three events due to antineutrino interactions have an associated $\mathrm{V}^{\circ}$. All the $\mathrm{V}^{\circ}$ 's were found during the general scan, before the special search for prompt electrons and before EMI information was available. Seven $\mathrm{V}^{\mathrm{O}} \mathrm{s}$ fit a $\mathrm{K}_{\mathrm{s}}^{\mathrm{O}}$, one is ambiguous between $\mathrm{K}_{\mathrm{s}}^{\mathrm{O}}$ and $\Lambda$, one is ambiguous between decay and interaction of a neutral hadron (events 5 and 6 in table 1). For the $\mathrm{K}^{\circ}$ rates, quoted in table 2, the two ambiguous $\mathrm{V}^{\mathrm{O}}$ 's have each been counted as $0.5 \mathrm{~K}_{\mathrm{s}}^{\mathrm{O}}$ and the total sample has been corrected for the unseen neutral decay of $K_{S}^{0}$, for $K_{L}^{O}$, and for losses (10\%) due to decays at short distances and interactions. The corresponding $\mathrm{K}^{\circ}$ rates in ordinary neutrino and antineutrino $\mathrm{CC}$ events are found to be $0.16 \pm 0.02$ and $0.13 \pm 0.04$, respectively, i.e. a factor of about 10 lower than the $K^{\circ}$ rate in di-lepton events.

The $\mu^{-} \mathrm{e}^{+} / \mu^{-}$rates are consistent with the rates observed in two FNAL $15^{\prime}$ bubble chamber experiments: $(0.77 \pm 0.3) \%$ in light neon [3(a)] and 
$(0.5 \pm 0.15) \%$ in heavy neon [3(b)] for average event energies of 55 and $41 \mathrm{GeV}$, respectively; however, the measured $\mathrm{K}^{\circ}$ multiplicity in di-lepton events agrees with the former $(1.84+0.63)$ rather than with the latter $(0.5 \pm 0.2)^{(*)}$.

As found in previous experiments [1-4] the qualitative features of the di-lepton events favour an interpretation in which the first (rightsign) muon is from a charged current interaction and the second lepton is from the decay of a charmed particle. Simple charmed particle models predict a $\mathrm{K}^{\circ}$ multiplicity of about 0.8 [7], while $1.7 \pm 0.7$ is observed.

The data were also examined for heavy lepton production:

(i) In fig. 1, the momentum $p_{1}$ of the right sign muon is plotted against $\mathrm{p}_{2}$, the momentum of the second lepton. The lepton momentum asymmetry in the di-lepton events of the neutrino ex- . posure is $\left\langle p_{1}\right\rangle /\left\langle p_{2}\right\rangle=3.9 \pm 1.1$, which is outside the bounds for (spin $\frac{1}{2}$ ) heavy lepton decay $[8]$.

(ii) The vertex regions of all events have been examined for gaps between a possible di-lepton vertex and the neutrino event vertex. Within the spatial resolution (3-10 mopending on the final state multiplicity), the leptons in all sixteen events originate from the main event vertex. Hence, there is no evidence for the production of neutral long Iived heavy leptons as reported in ref. [9].

(iii) In the neutrino exposure the observed number of $\mathrm{e}^{-}$events with no muons is 16 , which after correction for identification inefficiency and background, becomes $24 \pm 6$; this is comparable with the rate of 26 events expected from the $\nu_{e}$ component of the beam due to $\mathrm{K}_{\mathrm{e} 3}^{+}$and $\mathrm{\mu}^{+}$decays. From this observation an upper limit can be deduced for the ratio

$$
\frac{v_{\mu} \rightarrow \tau}{v_{\mu} \rightarrow \mu}
$$

(*) The $\mathrm{K}^{0}$ rate deduced from ref. [3(b)] would predict $2.1 \pm 0.8$ observed $\mathrm{K}_{S}^{\mathrm{O}} \rightarrow \pi^{+} \pi^{-}$for this experiment; the probability to observe 7 or more, is less than $2 \%$. 
of $6 \%\left(90 \% \mathrm{CL}\right.$ ) assuming a $15 \%$ branching ratio for $\tau \rightarrow \mathrm{e} \nu_{\mathrm{e}} \nu_{\tau}$, where $\tau$ is the heavy lepton observed in $\mathrm{e}^{+} \mathrm{e}^{-}$interactions [10]. This result also applies for any other type of heavy lepton having a decay mode $\mathrm{L}^{-} \rightarrow \mathrm{e}^{-} \nu_{\mathrm{e}} \nu_{\mathrm{L}}$.

We would like to thank the scanning, measuring and computing staff at each of our laboratories for their work and express our indebtedness to our colleagues of the BEBC, EMI, SPS and beam groups. 
CERN/EP/PHYS 77-56

\section{REFERENCES}

[1] A. Benvenuti et al., Phys. Rev. Lett. 34 (1975) 419; 35 (1975) 1199, 1203 and 1249; 38 (1977) 113;

B.C. Barish et al., Phys. Rev. Lett. 36 (1976) 939; 38 (1977) 577.

[2] J. B1ietschau et a1., Phys. Lett. 58B (1975) 361; 60B (1976) 207.

[3] (a) J, von Krogh et a1., Phys. Rev. Lett. 36 (1976) 710; P.C. Bosetti et al., Phys. Rev. Lett. 38 (1977) 1248.

(b) C. Baltay et a1., Phys. Rev. Lett. 39 (1977) 62.

[4] M. Holder et a1., Phys. Lett. 69B (1977) 377; 70B (1977) 396 and 393.

[5] C. Brand et al., Nuc1. Instr. and Methods 136 (1976) 485.

[6] P.C. Bosetti et a1., Phys. Lett. 70B (1977) 273.

[7] M.K. Gaillard, Proceedings of the 1976 Aachen Neutrino Conference, p. 553.

[8] A. Pais and S. Treiman, Phys. Rev. Lett. 35 (1975) 1206.

[9] D.S. Baranov et al., Serpukhov preprint IFVE 77-30 (1977).

[10] M.L. Perl et a1., Phys. Rev. Lett. 35 (1975) 1489. 


\section{TABLE CAPTIONS}

Table 1 Characteristics of multi-lepton events. The quantities $x_{v i s}=q^{2} / 2 \mathrm{ME}_{\mathrm{H}}, \mathrm{y}_{v i \mathrm{~s}}=\mathrm{E}_{\mathrm{H}} / \mathrm{E}_{\text {vis }}$ are based on the measured hadron energy $E_{H}$ and total energy $E_{v i s}=E_{H}+E_{\mu} \Delta \phi$ is the difference of lepton azimuths around the neutrino beam direction. $\mathrm{p}_{2 \perp \mu \nu}$ is the momentum of the second lepton perpendicular to the $\mu \nu$-plane. No $\mu^{-} e^{-}$event was found.

Table 2 Relative di-lepton event rates. 
CERN/EP/PHYS 77-56

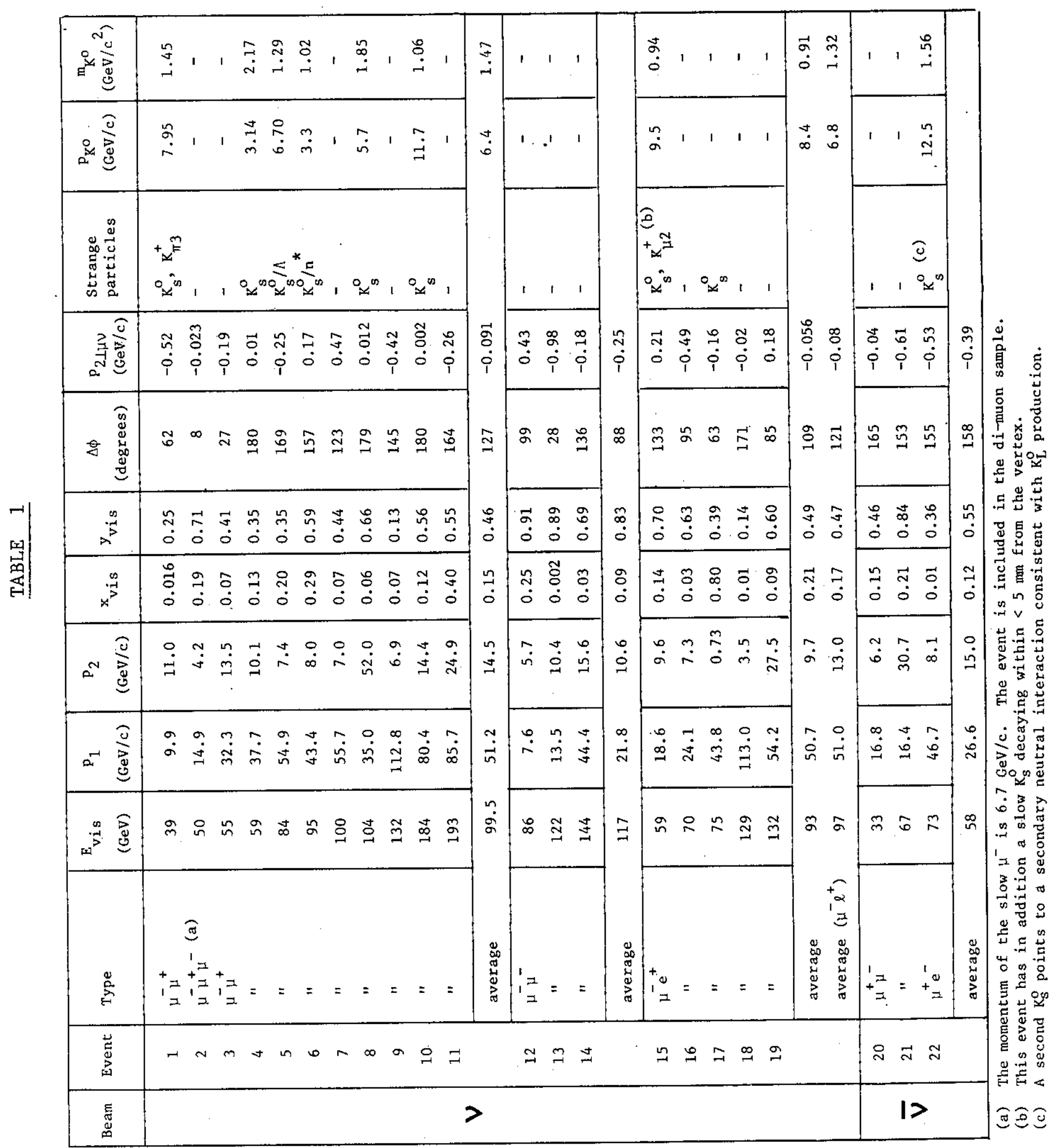


CERN/EP/PHYS 77-56

\begin{tabular}{|c|c|c|c|}
\hline 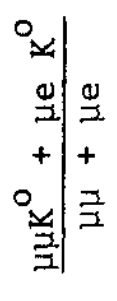 & 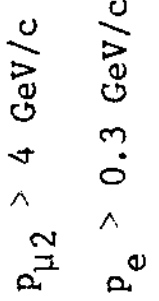 & $\begin{array}{l}\hat{0} \\
+1 \\
\tilde{H}\end{array}$ & $\begin{array}{l}0 \\
\dot{-1} \\
+1 \\
0 \\
\dot{0}\end{array}$ \\
\hline $\begin{array}{l}\text { No } \\
0 \\
+ \\
2 \\
3\end{array} \mid=$ & $\begin{array}{l}\frac{0}{3} \\
0 \\
\Delta \\
\hat{a}\end{array}$ & $\begin{array}{l}+ \\
0 \\
+1 \\
\overrightarrow{0} \\
\dot{0}\end{array}$ & $\begin{array}{c}\tilde{0} \\
+1 \\
\sim \\
\tilde{-}\end{array}$ \\
\hline \multirow{2}{*}{$\stackrel{\circ}{\beth}$} & $\begin{array}{l}0 \\
ن \\
0 \\
0 \\
\dot{j} \\
\wedge \\
0^{0}\end{array}$ & $\begin{array}{l}\overrightarrow{0} \\
\dot{0} \\
+1 \\
\stackrel{0}{0}\end{array}$ & \multirow{2}{*}{$\begin{array}{l}0 \\
0 \\
+1 \\
0 \\
0\end{array}$} \\
\hline & $\begin{array}{l}\frac{0}{\vec{d}} \\
\tilde{0} \\
\dot{0} \\
\hat{a}\end{array}$ & $\begin{array}{l}3 \\
\dot{0} \\
+1 \\
\ddot{0}\end{array}$ & \\
\hline \multirow[t]{2}{*}{ 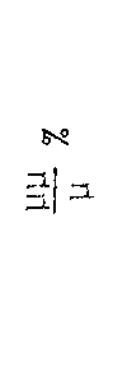 } & \multirow[t]{2}{*}{ 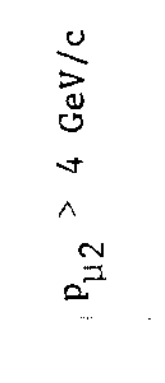 } & $\begin{array}{l}\mathfrak{0} \\
\dot{0} \\
+1 \\
\infty \\
\dot{0}\end{array}$ & $\begin{array}{l}0 \\
\dot{0} \\
+1 \\
\infty \\
\dot{0}\end{array}$ \\
\hline & & $>$ & 17 \\
\hline
\end{tabular}




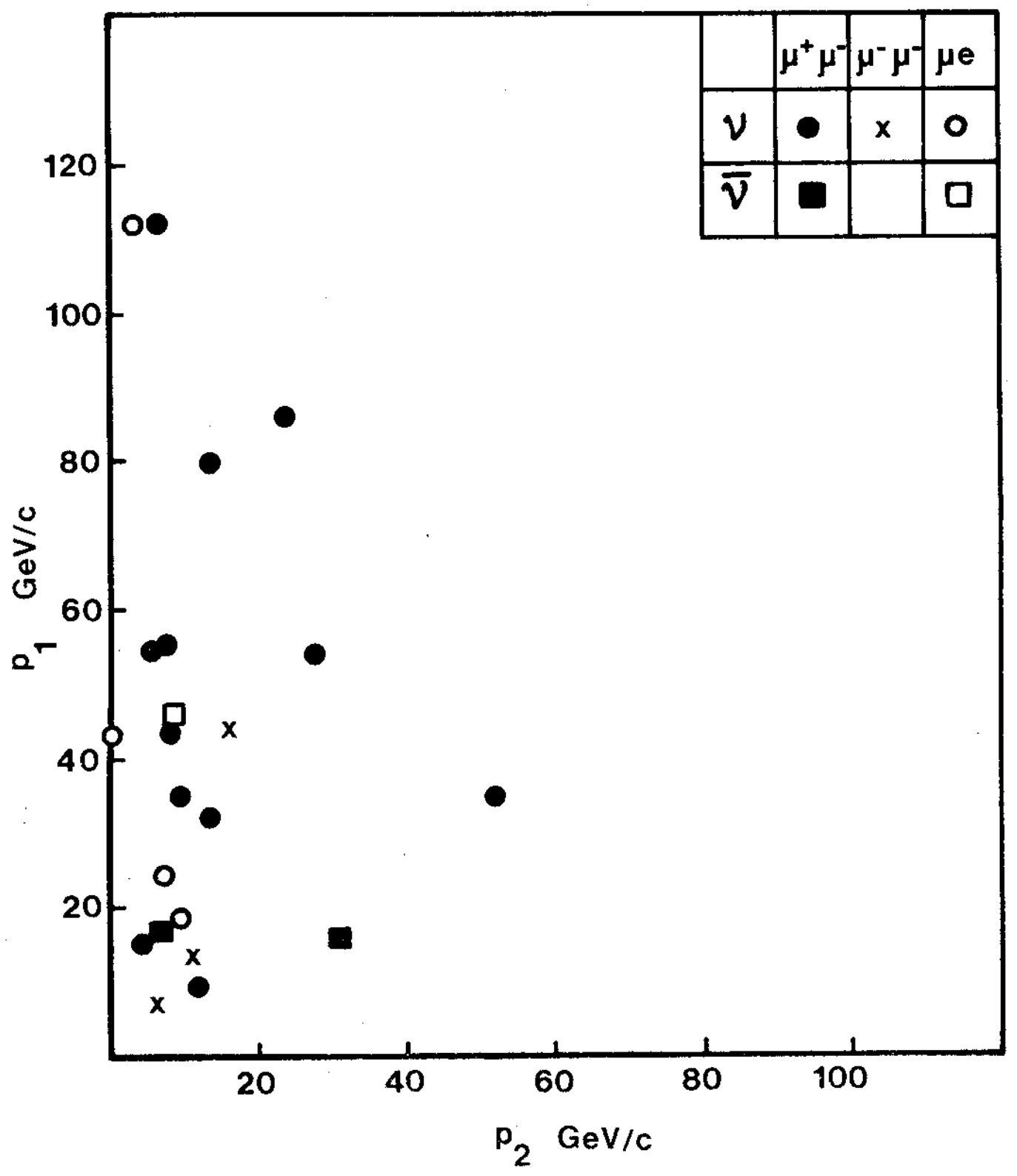

Fig. I Momentum of the right-sign muon, $p_{1}$, versus momentum of the second lepton, $\mathrm{p}_{2}$. 
4 